Review Article

\title{
Acute Kidney Injury in Children
}

\author{
Nilzete Liberato Bresolin,, ${ }^{1 *}$ Maria Goretti Moreira Guimarães Penido, ${ }^{2}$ Rejane de Paula Bernardes ${ }^{3}$ \\ ${ }^{1}$ Adjunct Professor of Pediatric Nephrology Federal University of Santa Catarina, Brazil
}

${ }^{2}$ Head pf Pediatric Nephrology Unit, Nephrology Center of Santa Casa de Belo Horizonte Hospital and Associate Professor of Pediatrics and Pediatric Nephrology Retired of Federal University of Minas Gerais, Brazil

${ }^{3}$ Director of the Nefrokids Clinic, Curitiba, Brazil

\begin{abstract}
Recent literature data has shown that acute kidney injury (AKI) is a common occurrence in critically ill children and that it is associated with high rates of morbidity and mortality, even after adjusting for other risk factors. In addition, it can result in long-term sequelae translated by the development of arterial hypertension, microalbuminuria and chronic renal dysfunction. High degree of suspicion, early diagnosis based on the recognition of patients at risk and the use of new criteria for the diagnosis and classification of AKI and, whenever possible, using the new biomarkers can positively alter the prognosis of these patients. Although there is still no specific treatment for AKI, it is recommended to focus on the immediate institution of preventive measures in order to maintain renal hemodynamics, early treatment of septic patients, elimination of exogenous nephrotoxins, recognition and management of patients with AKI secondary to high levels of endotoxins, control and normalization of volume after the initial phase of fluid resuscitation, sufficient nutritional support, and recognition and treatment of the underlying causes are necessary. Patients that fail with conservative care will need renal replacement therapy.
\end{abstract}

Keywords: Acute kidney injury, Nephrotoxicity, Hemoglobinuria, Endotoxins

\section{Introduction}

AKI commonly occurs in critically ill children, and its incidence has increased globally in recent years. Evidence demonstrates a significant association between AKI and morbidity and mortality in the acute phase and an association with long-term nephrological sequelae. ${ }^{1}$ For the past 15 years, advances in research on AKI have allowed us to recognize that even small renal function changes, previously considered insignificant, may negatively impact. Shortterm impact on length of stay and mortality and development of long-term renal dysfunction with microalbuminuria, proteinuria, arterial hypertension and impaired renal function. ${ }^{1}$ Mortality rates remain relatively high and range from $10 \%$ uncomplicated cases to $80 \%$ complicated cases requiring renal replacement therapy. ${ }^{2,3}$ In epidemiological terms, the prospective observational "AWARE" study, carried out in 2014, including children aged 3 to 25 years admitted to 32 intensive care units (ICU) in Asia, Australia, Europe, and North America, demonstrated a global incidence of $26.9 \%$ AKI among 4,683 children, $11.6 \%$ were considered with severe AKI (KDIGO 2 or 3). Patients with severe AKI had an odds ratio adjusted for mortality of 1.77 (CI 1.17-2.68) and a mortality rate of $11 \%$ versus $2.5 \%(p<0.001)$ for patients without severe AKI. ${ }^{4}$

Since the kidney is the central mediator of host homeostasis, the aberrant renal function will impact systemic health. Recent findings in the literature demonstrate that in addition to the obvious consequences associated with AKI, such as slag accumulation, hydro electrolytic, and acid-base disorders, some other effects are less apparent. AKI may also be involved with prognostic factors as impaired immunity, multiple organ dysfunctions (cross-talk), and a high degree of fluid overload (FO)., ${ }^{3,5}$ Unquestionably, monitoring, recognizing patients at risk for AKI, early diagnosis, and investigation are essential to reverse the kidney injury process and institute therapeutic measures to protect and prevent new injuries. ${ }^{6}$ In this context, the importance of early diagnosis can be exemplified in a

\begin{tabular}{|l|l|}
\hline Quick Response Code: & *Corresponding author: Nilzete Liberato Bresolin, Adjunct Professor of Pediatric Nephrolo- \\
gy Federal University of Santa Catarina, Brazil \\
Received: 05 February, 2021 \\
Citation: Bresolin NL, Penido MGMG, Bernardes RP. Acute Kidney Injury in Children. SOJ \\
Pedia Clin Neonato. 2021;1(2):1-8. DOI: 10.53902/SOJPCN.2021.01.000507
\end{tabular}


study by Sood et al.,7 This study included 5,000 patients from 28 services in Canada, the USA, and Saudi Arabia demonstrated that the improvement or even reversal of AKI during the first 24 hours has a significant association with a better prognosis. The same conclusion reported in a recently published meta-analysis that included eight studies on AKI prognosis and duration demonstrated an increased relative risk (RR) of death for patients with longer AKI duration, regardless of the KDIGO stage of AKI classification. ${ }^{8}$ In patients with a period less than or equal to two days, the RR of death was 1.42 (95\% CI 1.21-1.66), lasting from 3 to 6 days RR 1.92 (95\% CI $1.34-2.75$ ) and duration greater than or equal to 7 days RR 2.28, (95\% CI 1.77-2.94). The authors concluded that the longer duration of AKI is associated with mortality and cardiovascular risk and evolution to chronic renal dysfunction. ${ }^{8}$

\section{Suspect the occurrence of AKI}

What does it mean "suspect" for the occurrence of the AKI? Suspecting should be translated as developing a high degree of suspicion for the possibility of AKI since the clinical picture of AKI is, in most cases, highly nonspecific and usually multifactorial. ${ }^{1,2} \mathrm{~A}$ complete anamnesis and physical examination are essential. Countless conditions can result in AKI, such as different types of shock, sepsis, oncological complications, postoperative of cardiac surgery and major surgeries, significant burns, nephrotoxicity, and systemic diseases. Less frequent causes of AKI in children are vasculitis, accidents with venomous animals; renal impairment secondary to cross-talk between heart, lung, brain, intestine, liver, and kidneys; water overload; and an excess filtered load of endogenous substances as uric acid, myoglobin, and hemoglobin. Anoxia in newborns (NB), especially premature and very low birth weight, is highly prone to AKI due to renal immaturity. Babies with perinatal asphyxia are a high-risk group for AKI affects up to $60 \%$ of these patients because the kidneys are susceptible to oxygen deprivation. After hypoxemia, vasoconstriction mediated by adenosine release and decreased glomerular filtration rate (GFR) occurs. Adenosine receptor antagonists can inhibit this effect (e.g., theophylline administered in the first hour of life). ${ }^{9}$ Concerning cross-talk between organs, which addresses the fact that the impairment of a given organ can affect distant organs from multiple inflammatory pathways and increased expression of inflammatory mediators, the possibility of two-way should be noted. ${ }^{10}$

Cardiac impairment may be secondary to renal impairment (acute or chronic) and vice versa. Renal impairment can affect the lung, brain, and intestines. The liver and brain impairment (traumatic brain injury) can result in AKI, in the same way that induced fan barotrauma can release cytokines such as interleukins 1,6 and 8 in addition to tumor necrosis factor (TNF) and cause renal tubular cell apoptosis. ${ }^{11}$ Common intestinal changes in septic patients can also trigger and aggravate AKI-induced sepsis. ${ }^{12}$ Another critical risk factor for AKI is FO, commonly seen in critically ill patients. FO can be a clue to the diagnosis of AKI and can also be a cause of AKI because the kidney is an encapsulated organ with limited capacity to accommodate excess fluid. If there is FO, there will be an increase in renal venous pressure, renal interstitial edema, an increase in intra-abdominal pressure, a reduction in the glomerular filtration gradient and renal plasma flow and, consequently, a decrease in GFR with diuretic resistance and oliguria, worsening the FO due to an increased supply than a loss in a vicious cycle. ${ }^{13}$ Other significant factors are an excess of endogenous filtered load substances such hyperuricosuria in tumor lysis syndrome (SLT), hemoglobinuria in patients with massive hemolysis (e.g., secondary to extracorporeal circulation), myoglobinuria secondary to rhabdomyolysis by muscle crushing; viral infections (e.g., by COVID-19), and electrolyte disturbances ( $\mathrm{P}$ and $\mathrm{K}) \cdot{ }^{13-15}$

To exemplify the importance of surveillance, take a look at this case: a 14-year-old boy suffered a head trauma while running on a KART. He convulsed on the spot and was treated by Emergency Mobile Care Service and medicated with a benzodiazepine. Then he was transferred to the emergency room, where he arrived and presented another seizure crisis. He was treated and medicated with benzodiazepine and an attack dose of phenytoin. He received the entire care protocol for multiple trauma patients, including laboratory and imaging exams, and was referred to the intensive care unit (ICU) for monitoring (Glasgow coma scale of 7). Cranial computed tomography and abdominal ultrasound were normal. In the ICU, he presented stable vital data and urine output of $1.2 \mathrm{ml} / \mathrm{Kg} /$ hour. Twelve hours after admission, the creatinine was $1.7 \mathrm{mg} / \mathrm{dL}$. After 36 hours of admission, creatinine of $3.4 \mathrm{mg} / \mathrm{dl}$ and urea of 55 $\mathrm{mg} / \mathrm{dl}$. He had AKI according to pRIFLE (66.2\% reduction in clearance). After ruling out other causes of AKI, the possibility of acute tubulointerstitial nephritis was raised; phenytoin was suspended, and pulse therapy with methylprednisolone was promptly started. After the first pulse, a decline in creatinine and urea levels has already been observed; 48 hours later, serum creatinine was $2.2 \mathrm{mg} /$ dL. Thirty days later, the patient was discharged from the hospital, in good general condition, and with renal function restored only with conservative treatment for AKI. If no diagnosis and treatment were made, its evolution could be complicated, for example, by the need to institute renal replacement therapy (RRT). ${ }^{16,17}$

\section{Early Diagnosis}

The considerations described above reinforce the importance of surveillance for the possibility of AKI, which the search for early diagnosis must accompany. Traditionally, the diagnosis of AKI is made based on functional markers: reduced diuresis and altered serum creatinine, both a late consequence of AKI. Specifically concerning oliguria, although it is a good clue to the possibility of AKI, one should not wait for oliguria to make the diagnosis. AKI can present with normal, reduced, or even increased urinary output and, specifically, in cases secondary to nephrotoxicity and neonates that may have non-oliguric AKI. The urinary output is influenced by hydration status, diuretics, vasoactive amines, and antidiuretic hormone secretion in stressful situations. In turn, creatinine is a marker of low sensitivity. It needs time to accumulate before an increase in serum levels is observed. Ten to forty percent of its values are eliminated by tubular secretion, which can mask the initial reduction in the GFR. Their levels may not rise until 25 to $50 \%$ of kidney function has been lost. ${ }^{4}$ 
Furthermore, their generation depends on muscle mass, liver function, and meat supply in the diet. Daily 1 to $2 \%$ of muscle creatine transforms into creatinine at the liver. These factors are responsible for variations observed between males and females, between black and white/yellow, vegetarians and non-vegetarians, bodybuilders, and sedentary individuals. In critically ill patients, the serum creatinine levels may be falsely reduced in malnourished patients with muscle hypotrophy, in patients with myopathies, in individuals who have suffered limb amputation, and liver disease patients. Also, when we analyze serum creatinine levels, we do so within a particular volume of distribution. In hypervolemia situations, not uncommon after fluid resuscitation in critically ill patients, their levels may be falsely low due to dilution, and the child may have AKI with low serum creatinine levels1.In neonates, in the first three days of life, the creatinine serum levels reflect maternal creatinine. At birth, creatinine levels vary according to gestational age, and it is necessary to consult normal values (Table 1).1,18

Table 1: Values of plasma creatinine at birth vs gestational age (GA). ${ }^{18}$

\begin{tabular}{|c|c|}
\hline IG (weeks) & Creatinine $\mathbf{~} \mathbf{g} / \mathbf{d L}$ ( $(\mathbf{m} \mathbf{m o l} / \mathbf{L})$ \\
\hline 23 to 26 & 0.77 to $1.05(68.1$ to 92.8$)$ \\
\hline 27 to 29 & 0.76 to $1.02(67.2$ to 90.2$)$ \\
\hline 30 to 32 & 0.70 to $0.80(61.9$ to 70.7$)$ \\
\hline 33 to 45 & 0.77 to $0.90(68.1$ to 79.6$)$ \\
\hline
\end{tabular}

Despite all limitations of creatinine and urine output, it is important to note that in 2012 the new criteria for the diagnosis and classification of AKI, called KDIGO (Kidney Disease Improving Global Outcomes) (Table 2), ${ }^{1,19}$ were published to improve the overall results of kidney diseases. According to these criteria, based on variations in serum creatinine levels and/or in urinary output (the worst of the two criteria), patients (adults and children) with AKI are classified into three increasing renal dysfunction levels. Newborns criteria were validated in 2014, with two stage 1 modifications: increases greater than or equal to 0.3 in baseline creatinine or increases in baseline creatinine up to 1.9 then also include urine output between 0.5 and $1 \mathrm{ml} / \mathrm{Kg} /$ hour, which differs from the parameter for children and adults which is $<0.5 \mathrm{ml} / \mathrm{Kg} /$ hour. Also, about the definition of stage 3 , in older children and adults, it is considered 3 times increase in baseline creatinine or creatinine acutely greater than $4.0 \mathrm{mg} / \mathrm{dL}$, and in neonates, an increase of 3 times in basal creatinine or creatinine acutely greater than $2.5 \mathrm{mg} /$ $\mathrm{dL}$ (Table 3). ${ }^{20,21}$ Serial dosage of creatinine and continuous monitoring of diuresis is recommended. Besides urinalysis, must request the complete blood count, electrolyte dosage, blood gas analysis, and coagulogram. Imaging exams should include chest X-ray, ultrasound of kidneys and urinary tract, and echocardiogram. Additional tests should be ordered according to the suspected diagnosis (serumcomplement, protein electrophoresis, anti-nucleus factor, LE cells, ADAMTS13, renal biopsy).

Table 2: Classification of acute kidney injury (Kidney Disease Improving Global Outcomes - KDIGO). ${ }^{19}$

\begin{tabular}{|c|c|c|}
\hline AKI Stages & Serum creatinine (SCr) & Diuresis \\
\hline 1 & $\nabla 0.3 \mathrm{mg} / \mathrm{dl}$ or $>150-200 \%$ of baseline ( 1.5 to 2 times) & $<0.5 \mathrm{ml} / \mathrm{Kg} / \mathrm{h}$ for 6 hours \\
\hline 2 & $>200-300 \%$ of baseline (>2 - 3 times) & $<0.5 \mathrm{ml} / \mathrm{Kg} / \mathrm{h}>12$ hours \\
\hline 3 & $\begin{array}{c}>300 \% \text { of baseline ( }>3 \text { times or } \mathrm{SCr}>4.0 \mathrm{mg} / \mathrm{dL} \text { with sudden } \bowtie \text { of } \\
\text { at least } 0.5 \mathrm{mg} / \mathrm{dL} \text { ) }\end{array}$ & $<0.3 \mathrm{ml} / \mathrm{Kg} / \mathrm{h}$ for 24 hours or anuria for 12 hours \\
\hline
\end{tabular}

Table 3: Classification of Neonatal Acute Kidney Injury (Kidney Disease Improving Global Outcomes - KDIGO).20,21

\begin{tabular}{|c|c|}
\hline AKI Stages & Definition \\
\hline 0 & No significant change in creatinine \\
\hline 1 & $\otimes S C r$ by $0.3 \mathrm{mg} / \mathrm{dL}$ within $48 \mathrm{~h}$ or $\otimes$ in $\mathrm{SCr}$ by $150 \%$ to $<200 \%$ from previous trough \\
\hline 2 & \in SCr by $200 \%$ to $<300 \%$ of previous trough \\
\hline 3 & 『in SCr囚300\% of previous trough or SCr『2.5 mg/dL or RRT \\
\hline
\end{tabular}

SCr, serum creatinine

The various limitations associated with creatinine levels and urine output have stimulated the search for new structural biomarkers (BM) that allow an earlier AKI diagnosis. Several new BMs are being studied, and some are very promising. However, still limited by high cost and because they are not widely available. Among these, we have the BM of glomerular function (cystatin C), BM of damage and renal tubular repair, BM of inflammation, and BM of stress stand out. Cystatin C (Cys-C) is a protein synthesized by all nucleated cells, freely filtered by the glomeruli and, almost wholly, reabsorbed and catabolized by proximal tubular cells. Concerning creatinine, it has the advantage that its elevated serum levels directly reflect the fetal and infant glomerular filtration rate (GFR) because the placenta does not filter this protein and, in a situation of impaired glomerular filtration, it accumulates rapidly. ${ }^{22}$ In addition to Cys-C, proteomic analyses demonstrate that lipocalin associated with neutrophil gelatinase (NGAL) is one of the most highly induced proteins in the distal nephron segments after ischemic or nephrotoxic aggression in animals models. ${ }^{22}$ The urinary (NGAL) has activity antimicrobial, participates in iron metabolism and inflammatory processes, and acts as a growth factor that positively regulates epithelial proliferation. In the face of tubular ischemia, there is a large accumulation of NGAL in the blood and urine as a response to limit tissue damage. ${ }^{23}$

Another tubular injury marker is kidney injury molecule 1 (KIM-1), a transmembrane glycoprotein undetectable in normal 
kidneys. However, in ischemic and nephrotoxic injury, it is observed that its levels are high in the apical membrane of proximal tubular cells, as it is associated with the promotion of epithelial repair and phagocytosis. There are few studies on the predictive ability of KIM-1 in premature infants with $\mathrm{AKI} .{ }^{23}$ In addition to glomerular filtration markers and tubular injury, there are also inflammatory markers. Among these, the interleukin-8 (IL-8) is a cytokine pro-inflammatory agent that acts as a mediator of proximal tubular injury and is present in urine after ischemic injury. Studies with asphyxiated RNT demonstrated that urinary levels of NGAL and IL18 on the first day of life are significantly higher in those who developed AKI and that elevated levels of NGAL on the first day were predictive of mortality in infants with hypoxic-ischemic encephalopathy23. However, despite the numerous studies on BMs, none of them are used routinely in clinical practice, and there are still many questions to be answered. In this context, Jetton and Askenazi ${ }^{24}$ recently stated that urinary and serum tests of NGAL and KIM-1 could be available for bedside use because they are undergoing testing and review by the US Food and Drug Administration (FDA).

More recently, a proteomic approach has made it possible to identify the so-called BM of stress, which are markers of interruption of the cell cycle: the Metalloproteinidase-2 Tissue Inhibitor (TIMP-2) and the insulin-like growth factor binding protein (IGFBP7) that are expressed in tubular cells and act through regulatory proteins p27 and p53 to interrupt G1 cell cycle. These BMs represent a defense mechanism to prevent the division of potentially damaged cells. ${ }^{1}$ In 2014, the FDA approved nephrochec (which measures the product of urinary TIMP-2 IGFBP-7 concentrations) as the first commercial test to predict AKI in critical adults. ${ }^{25}$ Westhoffet al. demonstrated in a prospective cohort with 133 patients aged between zero and eighteen years, undergoing cardiac surgery, that the IGFBP7 and TIMP-2 product was able to predict the occurrence of AKI before azotemia and oliguria $(p<0.01)$ and performed well in predicting the risk of death in 30 days and three months. Gocze et al., ${ }^{26}$ in turn, demonstrated in a prospective study that included 107 patients undergoing major surgery, non-cardiac, with a high risk of AKI (which occurred in $42 \%$ of cases) that the product IGFBP7 and TIMP-2 detected from significantly to patients at risk of AKI. ${ }^{27}$ The authors pointed out that due to their rapid elevation, they facilitate early therapeutic intervention. Chen et al. prospectively studied 237 neonates in the ICU, with urine samples collected correctly to measure IGFBP7 and TIMP-2, and concluded that they have a discriminative value for severe AKI in critically ill neonates. ${ }^{28}$

However, although recent evidence from clinical studies involving BM has shown that they are promising in preventing early diagnosis and AKI management, there are still undefined points to be clarified. There is a need for further studies to identify BMs that can be appropriately selected and identify high-risk patients and different AKI phenotypes. ${ }^{29}$ Moreover, in this context, two new diagnostic and prognostic tools have been presented in the last decade: the role of hemodynamic parameters translated by clinical indicators capable of predicting AKI occurrence and its evolution and the furosemide stress test (FST). Concerning clinical indicators, the FINNA-
KI study deserves mention, in which 423 adult patients with severe sepsis were included, of which 153 (36\%) progressed to AKI. The authors demonstrated that lower mean arterial pressure (MAP) levels showed an independent association for AKI progression. ${ }^{30}$

Other authors have demonstrated that higher levels of systemic oxygen transport and MAP are independently associated with less probability of progression from stage 1 to AKI stage $3 .^{31}$ Increased degrees of fluid overload and oliguria have also been presented as significant and early indicators for the possibility of AKI and adverse evolution. ${ }^{32,33}$ Regarding the FST, tubular integrity test, the same is done with administering $1.0 \mathrm{mg} / \mathrm{Kg}$ intravenous bolus in patients who suspect AKI and are not using diuretics, and $1.5 \mathrm{mg} /$ Kg intravenous bolus in patients using diuretics. Next, diuresis should be monitored for 2 hours. The test is unresponsive when diuresis is less than $100 \mathrm{ml} / \mathrm{h}$ in adults and less than $1.0 \mathrm{ml} / \mathrm{Kg} /$ hour in children. The non-responsive FST predicts progression to more advanced AKI stages with high sensitivity and specificity and helps decide the need for RRT. It can be useful to assist decision on starting RRT. ${ }^{34}$

\section{Prevention of AKI}

The steps for preventing AKI will now be addressed. It is essential to identify patients at risk and eliminate, whenever possible, predisposing factors. Intensive care is need for risk patients such as premature, septic, patients with chronic diseases (predominantly renal or cardiac), critical patients using nephrotoxic drugs, those undergoing contrasted exams, and victims of accidents with animals venomous, polytraumatized, postoperative cardiac surgery, or other major surgeries. Predisposing factors include hypovolemia, fluid overload, hypoxemia, hypotension, hypoperfusion, use of nephrotoxins. In this case, it is essential to restore renal hemodynamics by re-establishing blood volume, oxygenation, MAP, oxygen transport from volume administration, inotropes, vasopressors, and/or vasodilators according to the hemodynamic condition of each patient.

Recently, a system using electronic health records was implemented and demonstrated positive results in assisting doctors in the early detection of AKI and, thus, in reducing the influence of risk factors. ${ }^{35}$ This system quickly points out high-risk patients to the medical team, allowing preventive and therapeutic actions are improving the rates of recovery of AKI. In pediatric patients, a system has been developed to screen children with multiple exposures to nephrotoxins resulting in faster monitoring for the possibility of AKI. There was a positive effect in reducing multiple exposures to nephrotoxins and, ultimately, also to $\mathrm{AKI} .{ }^{36}$ In addition to exogenous nephrotoxins, there are also endogenous nephrotoxins, among which the following stand out: hemoglobinuria, which is joint postoperative cardiac surgery with cardiopulmonary bypass (CPB) and after transfusion reactions; myoglobinuria secondary to crushing rhabdomyolysis, viruses, malignant hyperthermia and/or metabolic disorders of phosphorus and calcium and hyperuricosuria commonly associated with tumor lysis syndrome (TLS). These endotoxins can cause AKI by direct cellular toxicity, vasoconstriction 
resulting in hypoperfusion, and oxygen free radicals release. ${ }^{37,38}$ In most cases, kidney damage can be prevented by instituting measures such as hyperhydration and furosemide use (to improve tubular flow and to avoid obstruction) in patients who respond with diuresis. In hemoglobinuria cases, AKI appears to be associated with heme pigment changes when in contact with acidic urine. Hemoglobin dissociates, and there is evidence that hematin is a toxic pigment that, especially in volume depletion, may predispose to induced pigment AKI. ${ }^{37}$ In cases of TLS, the role of hyperuricemia in the development of AKI is well established. Hyperuricemia causes AKI by crystallization and precipitation in the renal tubules and also by independent crystal mechanisms. ${ }^{38}$

Regarding the treatment of hyperuricemia and its deleterious effects, it is essential to recognize that allopurinol, a xanthine oxidase inhibitor, inhibits its production. However, it does not affect the existing hyperuricemia, and therefore, it should be used preferably in patients with a low or intermediate risk of TLS. Rasburicasecatalyzes the enzymatic reduction of uric acid in allantoin (totally water-soluble) and, thus, quickly reduces the existing hyperuricemia. Its role in the treatment of established TLS is widely accepted, and there is clear evidence that its effectiveness is superior to the efficacy of allopurinol in reducing uric acid levels. ${ }^{37,38}$ Although urinary alkalinization has been recognized as part of the treatment of TLS with hyperuricosuria, facilitating urinary elimination of uric acid, recent data demonstrate that it should not be used routinely (except in cases of concomitant metabolic acidosis) due to the risk of calcium phosphate precipitation, which can also result in renal impairment. There is no evidence to recommend urinary alkalinization in AKI cases secondary to myoglobinuria (in patients with rhabdomyolysis). Here, it is worth highlighting the several reports of AKI secondary to myoglobinuria (due to rhabdomyolysis) in patients with COVID-19. ${ }^{39}$ Possible pathophysiological mechanisms include direct muscle invasion by viruses, cytokine storm resulting in muscle damage, and muscle damage by circulating viral toxins. Although there are no reports of muscle biopsies in these patients with rhabdomyolysis, it is known that COVID-19 infection is associated with elevated inflammatory markers, and this may indicate that cytokines may mediate rhabdomyolysis in COVID-19. ${ }^{39}$ Still, regarding prevention, there are patients in the postoperative period (PO) of cardiac surgery for whom, as demonstrated in several studies, AKI is recognized as one of the most potent prognostic factors. ${ }^{40}$ It should not be only related to water retention and metabolic disorders (uremia, acidosis, hyperkalemia) and cardiopulmonary bypass, and the inflammatory reaction resulting in increased capillary permeability, generalized tissue edema, and myocardial dysfunction with decreased urinary output and increased mechanical ventilation time. With the longer duration of mechanical ventilation, there is a greater risk of barotrauma, infection, development of acute respiratory distress syndrome (ARDS), multiple organs and system dysfunction (DMOS), and increased mortality rate. ${ }^{40-45}$

The various studies on the subject formed the basis for the prophylactic indication of RRT in cardiac surgery PO to prevent fluid accumulation and reduce mortality. ${ }^{40}$ High-risk patients leave the operating room with a peritoneal dialysis catheter installed. In these patients, the guideline KDIGO recommends alkalinization with intravenous saline solution or sodium bicarbonate for patients at risk of AKI. ${ }^{19}$ A pilot study by Hasse et al. with urinary alkalinization also demonstrated a significant reduction in the risk of AKI in patients undergoing cardiac surgery with cardiopulmonary bypass. ${ }^{41}$ However, a systematic review that included 3,563 patients found no evidence of the benefits of sodium bicarbonate use. ${ }^{42} \mathrm{~A}$ recent meta-analysis also showed no association between infusion of sodium bicarbonate and reduced incidence of AKI. ${ }^{42,43}$ As previously mentioned, a population that should be considered high-risk for AKI is neonates with severe hypoxia. In these patients, due to perinatal asphyxia, renal vasoconstriction occurs secondary to adenosine metabolites that cause decreased GFR and filtration fraction. This effect can be inhibited by a nonspecific adenosine receptor antagonist, for example, theophylline..$^{9,19,44}$

\section{Treatment of AKI}

With the prevention considerations in mind, we will move on to the therapeutic approach itself. There is no curative treatment for AKI, and, at the moment, most of the strategies validated in the literature focus on prevention and minimization of renal dysfunction. ${ }^{1}$ These strategies include diagnosing and treating the underlying disease and renal homeostasis maintenance until renal function recovery and prevention of new injuries. The treatment of AKI is carried out with conservative measures and, whenever necessary, with RRT. Conservative treatment includes maintaining hydro-electrolytic and acid-base homeostasis with water balance control. ${ }^{45,46}$ Weight control and diuresis are essential to ensure cardiac output and good mean MAP from inotropes, vasopressors, and/or vasodilators according to the patient's hemodynamic condition. Regarding water supply in critically ill patients, the importance of early targeted therapy is unquestionable, with randomized studies demonstrating a better prognosis for patients in shock who receive early fluid, antibiotics, and oxygen therapy. ${ }^{19,37,45}$ Despite several reviews, the fluid dose to be administered during the critically ill patient's resuscitation remains highly empirical. Too little fluid can result in tissue hypoperfusion and worsening of organ dysfunction, and over-prescribing fluids can result in risks of negative impact on organ function. The negative effect of fluid overload (FO) on critically ill patients has become evident in recent years. Several studies have shown that FO exceeding values between 10 and $20 \%$ increases the relative risk of death by up to 3 times. In addition to increasing the risk of death, length of hospital stays even after corrections for demographic factors and the severity of the disease. . $^{13,45,46}$

Another crucial point in handling AKI is nutritional support. The nutritional restriction does not apply to AKI patients due to the risk of malnutrition and loss of body mass, and the risk of organ dysfunction and immune deficit. For critically ill children with AKI nutritional support should be individualized, suggesting the replacement of 120 to $130 \%$ of basal caloric needs and protein supply of 2 to $3 \mathrm{~g} / \mathrm{Kg} /$ day. ${ }^{46}$ RRT should be indicated whenever there is difficulty in adapting nutritional support due to difficulty maintaining water balance. 
The furosemide use is controversial because of the risks of ototoxicity, nephrotoxicity, nephrocalcinosis, and volume depletion. However, according to several authors, it is justified in patients with AKI who respond with diuresis. ${ }^{47}$ Among the possible advantages of furosemide, the following stand out: reduction of oxygen consumption in the external renal medulla due to inhibition of the counter-current mechanism in the ascending loop of Henle; increased tubular urinary flow, minimizing the risk of obstruction by cell debris, crystals, and debris; help in controlling hyperpotassemia and in water handling in patients who respond to its administration. ${ }^{45-48}$ Recently, the importance of using it has been demonstrated in studies on the TEF already mentioned in the part dealing with early diagnosis. Studies show high sensitivity and specificity concerning critically ill patients' diagnosis and evolutionary prognosis with AKI. $^{34,49}$

Another point to be noted is that, more recently, studies have shown a risk of developing diuretic resistance due to chronic use of furosemide, p. ex, in cardiac patients..$^{50}$ It should be suspected when there is a decrease in the diuretic response without worsening renal function, hemodynamic impairment, or reduced fluid supply. This condition appears to be associated, among other mechanisms, with hypertrophy of renal distal tubular cells due to the increase in distal tubular sodium concentration induced by the inhibition of the counter-current mechanism in the ascending Henle loop. Hypertrophy would be an attempt to compensate and increase sodium reabsorption at this location. ${ }^{50}$ This complication can be treated by concomitant administration of low doses of a diuretic with action on the distal tubule (e.g., hydrochlorothiazide). This drug can be indicated for the treatment and prevention of nephrocalcinosis, another complication induced using furosemide. ${ }^{48,50}$ Particularly important is to highlight that in patients with AKI, nephrotoxins must be avoided. Still, in the impossibility of being avoided, they must be adjusted according to the creatinine clearance in patients outside dialysis therapy or according to the dialysis in those in RRT. ${ }^{46}$ Here, we highlight the new electronic systems that use clinical records and electronically signal to the medical team that patients are at high risk for AKI. In the pediatric age group, these systems allow the screening of children using multiple nephrotoxic drugs and, thus, the establishment of preventive measures against the occurrence of $\mathrm{AKI}^{51}$

\section{Renal replacement therapy (RRT)}

Although many controversies are reported about how and when to start RRT, most authors agree that it should start at the slightest sign of its need. Indications include FO with severe arterial hypertension and/or pulmonary edema, FO in PO of cardiac surgery with a high risk of AKI, acid-base and electrolyte disorders that do not respond to conservative treatment, severe and/or progressive uremia, need to adapt the nutritional support. Recently, a publication by the American College of Critical Care Medicine reinforced the early indication of RRT in children and neonates. After initial fluid resuscitation, they presented FO greater than $10 \%$ and could not maintain water and hydro electrolytic balance ${ }^{52}$. There are several modalities for RRT, and its indication will depend on the objectives of dialysis therapy, advantages and disadvantages of each method, the professional's ability, and institutional availability. Peritoneal dialysis remains a good option, especially for young and premature children. Besides, it remains the method of choice in pediatric ICUs in countries with limited financial resources ${ }^{2,3,52}$ It has the following advantages: a technically simple, low-cost method easily performed in hemodynamically unstable patients. However, the removal of fluids and slag can occur slowly and unpredictably. In hemodialysis, removing toxins occurs quickly; however, vascular access can be a problem in young children. There is a risk of anticoagulation, and, in unstable patients, it can result in worsening of the hemodynamic condition. Continuous RRT methods have been presented as a modality of choice in developed countries due to their high degree of effectiveness. The disadvantages of continuous methods include the need for staff with specific training, the high costs, the need for adequate vascular access, and the "size" of the extracorporeal circuit. Technological advances have resulted in specific equipment for neonates with promising results for their dialysis treatment. ${ }^{46}$

\section{Conclusion}

In the universe of AKI in critically ill children with potential consequences on the evolutionary prognosis about morbidity and mortality, there is a need for us to be vigilant about the possibility of its occurrence. The markers of functional renal impairment, traditionally used (creatinine and urine output), can and should continue to be used and represent the basis of the new criteria for the diagnosis and classification of AKI called KDIGO. However, it is necessary to recognize the limitations of these markers. Assess creatinine levels taking into account the factors that may affect your dosage and the importance of regular checks. Measure urine output accurately and analyze the result considering the various factors that can influence it. By identifying patients at risk and using new diagnostic tools (structural BM, clinical indicators, FST), we can act in the early intervention window. That period in which there is a risk of kidney damage and/or initial kidney damage, in general, precedes the reduction in GFR, which is diagnosed by increasing creatinine and/ or reducing diuresis. Measures to prevent kidney damage need to be known and instituted early. Recent evidence from the literature demonstrates an unmistakable association between the longer duration of AKI and patients' prognosis in the short and long term. Although there are no specific medications for AKI already established, early preventive and therapeutic intervention is essential to improve these prognoses.

\section{Acknowledgments}

None.

\section{Funding}

None.

\section{Conflicts of Interest}

Michael J. Dochniak is co-founder and CTO of Alleam, LLC. Minnesota, USA. 


\section{References}

1. Ciccia E, Devarajan P. Pediatric acute kidney injury: prevalence, impact and management challenges. Int J Nephrol Renovasc Dis. 2017;10:77-84.

2. Bresolin N, Silva C, Halllal A, et al. Prognosis for children with acute kidney injury in the intensive care unit. Pediatr Nephrol. 2009;24(3):537-44.

3. Bresolin N, Bianchini AP, Hass CA. Pediatric acute kidney injury assessed by pRIFLE as a prognostic factor in the intensive care unit. Pediatr Nephrol. 2013;28(3):485-492.

4. Kaddourah A, Basu RK, Bagashaw SM, et al. Epidemiology of acute kidney injury in critically ill children and young adults. $N$ Engl J Med. 2017;376(1):11-20.

5. Askenazi D. Evaluation and management of critically ill children with acute kidney injury. Curr Opin Pediatr. 2011;23(2):201-7.

6. Bagshaw SM, Wald R. Strategies for the optimal timing to start renal replacement therapy in critically ill patients with acute kidney injury. Kidney Int. 2017;91(5):1022-32.

7. Sood MM, Shafer LA, Ho J, et al. Early reversible acute kidney injury is associated with improved survival in septic shock. J Crit Care. 2014;29(5):711-7.

8. Mehta S, Chauhan K, Patel A, et al. The prognostic importance of duration of AKI: a systematic review and meta-analysis. BMC Nephrol. 2018;19(1):91.

9. Bhatt GC, Gogia P, Bitzan M, et al. Theophylline and aminophylline for prevention of acute kidney injury in neonates and children: a systematic review. Arch Dis Child. 2019;104(7):670-9.

10. Dellepiane S, Marengo M, Cantaluppi V. Detrimental cross-talk between sepsis and acute kidney injury: new pathogenic mechanisms, early biomarkers and targeted therapies. Crit Care. 2016;15;20:61.

11. Khalid F, Yang GL, McGuire JL, et al. Autonomic dysfunction following traumatic brain injury: translational insights. Neurosurg Focus. 2019;47(5):E8.

12. Zhang J, Ankawi G, Sun J, et al. Gut-kidney crosstalk in septic acute kidney injury. Crit Care. 2018;22(1):117.

13. Romero OEN, Meléndez AFN. Fluid overload and kidney failure in children with severe sepsis and septic shock: A cohort study. Arch Argent Pediatr. 2017;115(2):118-24.

14. Naeem B, Moorani KN, Anjum M, et al. Tumor lysis syndrome in pediatric acute lymphoblastic leukemia at tertiary care center. Pak J Med Sci. 2019;35(4):899-904.

15. Taxbro K, Kahlow H, Wulcan H, et al. Rhabdomyolysis and acute kidney injury in severe COVID-19 infection. BMJ Case Rep. 2020;13(19):e237616.

16. Bresolin NL, Docusse Junior P, Shiozawa MBC, et al. Acute Tubulointerstitial Nephritis due to Phenytoin: Case Report. J Clini Nephrol. 2017;1:019-025.

17. Bresolin NL, Docusse Junior P. Acute tubulointerstitial nephritis due to phenytoin: Case report commentary. J Clin Nephrol Ther. 2018;2(1):123.

18. Ringer SA. Acute renal failure in the neonate. Neo Reviews. 2010;11(5):e243-251.

19. Kidney Disease: Improving Global Outcomes Acute Kidney Injury Work Group. KDIGO clinical practice guideline for acute kidney injury. Kidney Int. 2012;(Suppl)2:1-138.

20. Bakr A, Eid R, Allam NA, et al. Neonatal acute kidney injury: diagnostic and therapeutic challenges. J Nephrology Res. 2018;4(1):130-134.

21. Ricci Z, Ronco C. Neonatal RIFLE. Nephrol Dial Transplant. 2013;28(9):2211-2214.
22. Toda Y, Sugimoto K. AKI after pediatric cardiac surgery for congenital heart diseases-recent developments in diagnostic criteria and early diagnosis by biomarkers. J Intensive Care. 2017;5:49.

23. Marin T, De Rossett B, Bhatia J. Urinary Biomarkers to Predict Neonatal Acute Kidney Injury. J Perinat Neonat Nurs. 2018;32(3):266-274.

24. Jetton JG, Askenazi DJ. Update on acute kidney injury in the neonate. Curr Opin Pediatr. 2012;24(2):191-198.

25. Kellum JA, Chawla LS. Cell-cycle arrest and acute kidney injury: the light and the dark side. Nephrol Dial Transplant. 2016;31(1):16-22.

26. Westhoff JH, Tönshoff B, Waldherr S, et al. Urinary Tissue Inhibitor of Metalloproteinase-2 (TIMP-2) - Insulin-Like Growth Factor-Binding Protein 7 (IGFBP7) Predicts Adverse Outcome in Pediatric Acute Kidney Injury. PloS One. 2015;10(11):e0143628.

27. Gocze I, Koch M, Renner P, et al. Urinary biomarkers TIMP-2 anNIGFBP7 early predict acute kidney injury after major surgery. PLoS One. 2015;10(3):e0120863.

28. Chen J, Sun Y, Wang S, et al. The effectiveness of urinary TIMP-2 and IGFBP-7 in predicting acute kidney injury in critically ill neonates. Pediatr Res. 2020;87(6):1052-1059.

29. Ostermann M, Zarbock A, Goldstein S, et al. Recommendations on Acute Kidney Injury Biomarkers from the Acute Disease Quality Initiative Consensus Conference: A Consensus Statement. JAMA Netw Open. 2020;3(10):e2019209.

30. Poukkanen M, Wilkman E, Vaara ST, et al. Hemodynamic variables and progression of acute kidney injury in critically ill patients with severe sepsis: data from the prospective observational FINNAKI study. Crit Care. 2013;17(6):R295.

31. Uwaezuoke SN. Pediatric acute kidney injury: Appraisal of predictors and prognostic indicators. J Acute Dis. 2017;6(1):6-11.

32. Raimundo M, Crichton S, Martin JR Syed Y, et al. Increased Fluid Administration After Early Acute Kidney Injury is Associated with Less Renal Recovery. Shock. 2015;44(5):431-437.

33. Prowle JR, Liu YL, Licari E, et al. Oliguria as predictive biomarker of acute kidney injury in critically ill patients. Crit Care. 2011;15(4):R172.

34. Koyner JL, Davison DL, Brasha-Mitchell E, et al. Furosemide Stress Test and Biomarkers for the Prediction of AKI Severity. J Am Soc Nephrol. 2015;26(8):2023-2031.

35. Sutherland SM, Chawla LS, Kane-Gill SL, et al. Utilizing electronic health records to predict acute kidney injury risk and outcomes: workgroup statements from the 15(th) ADQI Consensus Conference. Can J Kidney Health Dis. 2016;26;3:11.

36. Goldstein SL, Kirkendall E, Nguyen H, et al. Electronic health record identification of nephrotoxin exposure and associated acute kidney injury. Pediatrics. 2013;132(3):e756-767.

37. Andreoli SP. Acute kidney injury in children. Pediatr Nephrol. 2009;24(2):253-263.

38. Alakel N, Middeke JM, Schetelig J, et al. Prevention and treatment of tumor lysis syndrome, and the efficacy and role of rasburicase. Onco Targets Ther. 2017;10:597-605.

39. Gefen AM, Palumbo N, Nathan SK, et al. Pediatric COVID-19-associated rhabdomyolysis: a case report. Pediatr Nephrol. 2020;35(8):1517-1520.

40. Blinder JJ, Goldstein SL, Lee VV, et al. Congenital heart surgery in infants: effects of acute kidney injury on outcomes. Thorac Cardiovasc Surg. 2012;143(2):368-374.

41. Haase M, Haase-Fielitz A, Bellomo R, et al. Sodium bicarbonate to prevent increases in serum creatinine after cardiac surgery: a pilot double-blind, randomized controlled trial. Crit Care Med. 2009;37(1):39-47.

42. Mei M, Zhao HW, Pan QG, et al. Efficacy of N-Acetylcysteine in preventing 
acute kidney injury after cardiac surgery: a meta-analysis study. I Invest Surg. 2018;31(1):14-23.

43. Zoungas S, Ninomiya T, Huxley R, et al. Systematic review: sodium bicarbonate treatment regimens for the prevention of contrast-induced nephropathy. Ann Intern Med. 2009;151(9):631-638.

44. Raina A, Pandita A, Harish R, et al. Treating perinatal asphyxia with theophylline at birth helps to reduce the severity of renal dysfunction in term neonates. Acta Paediatr. 2016;105(10):e448-51.

45. Schrier RW. Fluid administration in critically ill patients with acute kidney injury. Clin J Am Soc Nephrol. 2010;5:733-739.

46. Cho MH. Pediatric acute kidney injury: focusing on diagnosis and management. Child Kidney Dis. 2020;24:19-26.

47. Bagashaw SM, Bellomo R, Kellum JA. Oliguria, volume overload, and loop diuretics. Crit Care Med. 2008;36:S172-178.
48. Bestic M, Reed MD. Common diuretics used in the preterm and term infant. Neo Reviews. 2005;6:e392-8.

49. Elsaegh HK, Naga YS, Elsayed HM, et al. The role of furosemide stress test in the prediction of severity and outcome of sepsis-induced acute kidney injury. J Egypt Soc Nephrol Transplant. 2018;18(3):86-95.

50. Wilcox CS, Testani JM, Pitt B. Pathophysiology of Diuretic Resistance and Its Implications for the Management of Chronic Heart Failure. Hypertension. 2020;76(4):1045-1054.

51. Park S, Baek SH, Ahn S, et al. Impact of Electronic Acute Kidney Injury (AKI) Alerts with Automated Nephrologist Consultation on Detection and Severity of AKI: A Quality Improvement Study. Am J Kidney Dis. 2018;71(1):9-19.

52. Davis AL, Carcillo JA, Aneja RK, et al. American College of Critical Care Medicine clinical practice parameters for hemodynamic support of pediatric and neonatal septic shock. Crit Care Med. 2017;45(6):10611093. 